\title{
The weighted horizontal linear complementarity problem on a Euclidean Jordan algebra
}

\author{
Xiaoni Chi · M. Seetharama Gowda • \\ Jiyuan Tao
}

Received: date / Accepted: date

\begin{abstract}
A weighted complementarity problem ( ${ }_{\mathrm{wCP}}$ ) is to find a pair of vectors belonging to the intersection of a manifold and a cone such that the product of the vectors in a certain algebra equals a given weight vector. If the weight vector is zero, we get a complementarity problem. Examples of such problems include the Fisher market equilibrium problem and the linear programming and weighted centering problem. In this paper we consider the weighted horizontal linear complementarity problem (wHLCP) in the setting of Euclidean Jordan algebras and establish some existence and uniqueness results. For a pair of linear transformations on a Euclidean Jordan algebra, we introduce the concepts of $\mathbf{R}_{0}, \mathbf{R}$, and $\mathbf{P}$ properties and discuss the solvability of wHCLPs under nonzero (topological) degree conditions. A uniqueness result is stated in the setting of $\mathbb{R}^{n}$. We show how our results naturally lead to interior point systems.
\end{abstract}

Keywords Weighted horizontal linear complementarity problem · Euclidean Jordan algebra $\cdot$ Degree $\cdot \mathbf{R}_{0}$-pair

Mathematics Subject Classification (2010) 90C30

Xiaoni Chi

School of Mathematics and Computing Science, Guangxi Colleges and Universities Key Laboratory of Data Analysis and Computation, Guilin University of Electronic Technology, Guilin 541004, Guangxi, P. R. China

E-mail: chixiaoni@126.com

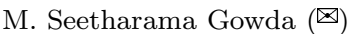

Department of Mathematics and Statistics, University of Maryland, Baltimore County, Baltimore, Maryland 21250, USA

E-mail: gowda@umbc.edu

Jiyuan Tao

Department of Mathematics and Statistics, Loyola University Maryland, Baltimore, Mary-

land 21210, USA

E-mail: jtao@loyola.edu 


\section{Introduction}

Introduced in 13, a weighted complementarity problem ( $\mathrm{wCP}$ ) is to find a pair of vectors $(x, y)$ belonging to the intersection of a manifold with a cone such that their product in a certain (Euclidean Jordan) algebra equals a given weight vector $w$. When $w$ is the zero vector, wCP becomes a complementarity problem (CP). To elaborate, consider a Euclidean Jordan algebra $(V, \circ,\langle\cdot, \cdot\rangle)$ with symmetric cone $V_{+}$[5]. Given a map $F: V \times V \times \mathbb{R}^{l} \rightarrow V \times \mathbb{R}^{l}$ and a weight vector $w \in V_{+}$, wCP is to find $(x, y) \in V \times V$ such that for some $u$,

$$
\begin{aligned}
& x \in V_{+}, y \in V_{+}, \\
& x \circ y=w, \\
& F(x, y, u)=0 .
\end{aligned}
$$

Deferring this general problem for a future study, in [13] and [14, Potra studies affine wCP on the (Euclidean Jordan) algebra $\mathbb{R}^{n}$ with several examples and results. Given matrices $A, B \in \mathbb{R}^{(n+m) \times n}, C \in \mathbb{R}^{(n+m) \times m}$, a weight vector $w \in \mathbb{R}_{+}^{n}$, and $q \in \mathbb{R}^{n+m}$, the weighted mixed horizontal linear complementarity problem considered in [13], [14] is to find $(x, y, z) \in \mathbb{R}^{n} \times \mathbb{R}^{n} \times \mathbb{R}^{m}$ such that

$$
\begin{aligned}
& x \geq 0, y \geq 0, \\
& x * y=w, \\
& A x+B y+C z=q,
\end{aligned}
$$

where $x * y$ denotes the Hadamard (= componentwise) product of vectors $x$ and $y$. Here, $C$ is assumed to be of full column rank and so, with a suitable change of variables, one could transform (see Section 2 in 14]) the above problem to an equivalent affine wCP where $C$ becomes vacuous and $A$ and $B$ are square. It is shown in 13 , that the Fisher market equilibrium problem [3], 17] and the linear programming and weighted centering problem [1] can be formulated in the form (2), where the triple $(A, B, C)$ satisfies a certain monotonicity condition. In 13, Potra presented and analyzed two interiorpoint methods for solving such monotone affine wCPs. Subsequently, replacing 'monotone' conditions by 'row and column sufficient' conditions, Potra [14] described several theoretical results and a corrector-predictor interior-point method for its numerical solution.

We note that weighted complementarity problems were studied much earlier in connection with interior point methods. For example, in [8, Kojima et al showed that if (continuous) $f: \mathbb{R}^{n} \rightarrow \mathbb{R}^{n}$ is a uniform P-function, that is, there exists some $\gamma>0$ such that

$$
\max _{1 \leq i \leq n}\left(x_{i}-y_{i}\right)\left(f_{i}(x)-f_{i}(y)\right) \geq \gamma\|x-y\|^{2}\left(\text { for all } x, y \in \mathbb{R}_{+}^{n}\right),
$$

then the mapping $\widehat{f}: \mathbb{R}_{+}^{n} \times \mathbb{R}_{+}^{n} \rightarrow \mathbb{R}_{+}^{n} \times \mathbb{R}^{n}$ is a homeomorphism, where

$$
\widehat{f}(x, y)=\left[\begin{array}{c}
x * y \\
y-f(x)
\end{array}\right] .
$$


In this situation, the following weighted nonlinear complementarity problem has a unique solution for each $w \in \mathbb{R}_{+}^{n}$ and $q \in \mathbb{R}^{n}$ :

$$
\begin{aligned}
& x \geq 0, y \geq 0, \\
& x * y=w, \\
& y=f(x)+q .
\end{aligned}
$$

Another work that specifically looks at the general wCP (1) is by Yoshise [18. In this work, under certain'monotonicity and injectivity' assumptions, it is shown that the map $(x, y, u) \rightarrow(x \circ y, F(x, y, u))$ is a homeomorphism on a certain subset of $V_{+} \times V_{+} \times \mathbb{R}^{l}$, leading to the solvability of wCP, see Theorem 3.10 and Corollary 4.4 in 18 .

Our objective in this paper is to consider the following affine ${ }_{\mathrm{wCP}}$ in the setting of Euclidean Jordan algebras. Given two linear transformations $A$ and $B$ on a Euclidean Jordan algebra $V$, a weight vector $w \in V_{+}$, and $q \in V$, the weighted horizontal linear complementarity problem $\operatorname{wHLCP}(A, B, w, q)$ is to find $(x, y) \in V \times V$ such that

$$
\begin{aligned}
& x \geq 0, y \geq 0, \\
& x \circ y=w, \\
& A x+B y=q,
\end{aligned}
$$

where $x \geq 0$ means that $x \in V_{+}$, etc. If $w=0$, the above problem reduces to the (symmetric cone) horizontal linear complementarity problem on $V$, denoted by $\operatorname{HLCP}(A, B, q)$. If $w=0, A=I$, and $B=-M, \operatorname{HLCP}(A, B, q)$ reduces to the (symmetric cone) linear complementarity problem $\operatorname{LCP}(M, q)$ on $V$. In particular, when $V=\mathbb{R}^{n}$, this reduces to the standard linear complementarity problem.

Our analysis differs from Potra's [13, [14 in several ways. First, our setting is that of a general Euclidean Jordan algebra, instead of $\mathbb{R}^{n}$. Second, instead of the 'monotone/sufficient' conditions, we rely on the $\mathbf{R}_{0}$ property (that is commonly used in the LCP literature) coupled with a nonzero degree condition of a certain map associated with wHLCP. Third, instead of using the optimization methodology, we rely on the degree theoretic tools. Our analysis also differs from that of Yoshise 18 where results were proved under certain 'monotonicity and injectivity' conditions.

In this paper, we establish some basic existence/uniqueness results about wHLCPs. Generalizing the LCP concept of a degree of an $\mathbf{R}_{0}$-matrix, we introduce the concept of degree of an $\mathbf{R}_{0}$-pair of linear transformations in the setting of Euclidean Jordan algebras. Assuming that this degree is nonzero for the pair $\{A, B\}$, we show that $\operatorname{wHLCP}(A, B, w, q)$ has a nonempty compact solution set for every $(w, q) \in V_{+} \times V$. This conclusion, in particular, will allow us to say that

- the map $\Gamma: V_{+} \times V_{+} \rightarrow V_{+} \times V$ given by $\Gamma(x, y)=(x \circ y, A x+B y)$ is surjective and 
- when $w>0$ (that is, $\left.w \in \operatorname{int}\left(V_{+}\right)\right)$, the 'interior point system'

$$
x>0, y>0, x \circ y=w, \text { and } A x+B y=q
$$

has a nonempty compact solution set.

The result about the interior point system appears to be new even in the setting of standard LCPs.

We also introduce the concept of a $\mathbf{P}$-pair and show that when $V=\mathbb{R}^{n}$, ${ }_{\mathrm{wHLCP}}(A, B, w, q)$ has a unique solution for every $(w, q) \in \mathbb{R}_{+}^{n} \times \mathbb{R}^{n}$.

The organization of our paper is as follows. In Section 2, we cover some basic material. In Section 3, we introduce the concepts of $\mathbf{R}_{0}$ and $\mathbf{R}$ pairs and define the degree of an $\mathbf{R}_{0}$-pair. Section 4 covers the main result of the paper describing the solvability of wHLCP. While Section 5 deals with the solution set behavior, Sections 6 and 7 cover $\mathbf{P}$-pairs and address uniqueness issues.

\section{Preliminaries}

Throughout this paper, $\mathbb{R}^{n}$ denotes the Euclidean $n$-space of real column vectors. We use the (same) symbol 0 to denote the zero vector in any vector space. $(V, \circ,\langle\cdot, \cdot\rangle)$ denotes a Euclidean Jordan algebra of rank $n$ with symmetric cone $V_{+}$[5], 7]. Here, $x \circ y$ and $\langle x, y\rangle$, respectively, denote the Jordan product and the inner product of elements $x$ and $y$. The unit element of $V$ is denoted by $e$. For a subset $S$ of $V$, the interior, closure, and boundary are denoted by $\operatorname{int}(S)$, $\bar{S}$, and $\partial(S)$. If $x \in V_{+}\left(x \in \operatorname{int}\left(V_{+}\right)\right)$, we write $x \geq 0$ (respectively, $x>0$ ). For $x \in V, x^{+}$denotes the projection of $x$ onto $V_{+}$, and we let $x^{-}:=x^{+}-x$, $|x|:=x^{+}+x^{-}$. These can also be described via the spectral decomposition $x=\sum_{1}^{n} x_{i} e_{i}$ (where $x_{1}, x_{2}, \ldots, x_{n}$ are the eigenvalues of $x$ and $\left\{e_{1}, e_{2}, \ldots, e_{n}\right\}$ is Jordan frame): $x^{+}=\sum_{1}^{n} x_{i}^{+} e_{i},|x|=\sum_{1}^{n}\left|x_{i}\right| e_{i}$, etc. We see that $|x|^{2}=x^{2}$, $\sqrt{x^{2}}=|x|,\left\langle x^{+}, x^{-}\right\rangle=0$ and $x^{+} \circ x^{-}=0$. For $x, y \in V$, we define

$$
x \sqcap y:=x-(x-y)^{+} .
$$

When $V=\mathbb{R}^{n}$ (with the usual componentwise product and the inner product), this reduces to $\min \{x, y\}$, the componentwise minimum of (vectors) $x$ and $y$ in $\mathbb{R}^{n}$. For this reason, we may call the map $(x, y) \rightarrow x \sqcap y$, the 'min map' on $V$. The map

$$
(x, y) \rightarrow x+y-\sqrt{x^{2}+y^{2}}
$$

is called the Fischer-Burmeister map. It has been extensively used in the complementarity literature. Below, we state some basic properties of these two maps.

Proposition 1 The following statements hold in $V$ :

(i) $u+x \sqcap y=(u+x) \sqcap(u+y)$.

(ii) $\lambda(x \sqcap y)=\lambda x \sqcap \lambda y$ for all $\lambda \geq 0$.

(iii) The following are equivalent: 
(a) $x \sqcap y=0$.

(b) $x \geq 0, y \geq 0$, and $\langle x, y\rangle=0$.

(c) $x \geq 0, y \geq 0$, and $x \circ y=0$.

Moreover, in each case, $x$ and $y$ operator commute.

(iv) When $w \geq 0$, the following are equivalent:

(a) $x+y-\sqrt{x^{2}+y^{2}+2 w}=0$

(b) $x \geq 0, y \geq 0$, and $x \circ y=w$.

Moreover, when $w=0$ or $w=e$ (the unit element of $V$ ), above $x$ and $y$ operator commute.

Proof. Items $(i)$ and (ii) follow easily from the definition of 'min map'. Item (iii) appears in [7, Proposition 6 and Item (iv) for $w=0$ or $w=e$ is covered in 7. Propositions 6 and 7. Now, let $w \geq 0$ and suppose $x+y-\sqrt{x^{2}+y^{2}+2 w}=$ 0 . Then, $x+y=\sqrt{x^{2}+y^{2}+2 w}$. This shows that $x+y \geq 0$ and (upon squaring and simplifying) $x \circ y=w$. We need only show that $x \geq 0$ and $y \geq 0$. Consider the spectral expansion $x=\lambda_{1} e_{1}+\lambda_{2} e_{2}+\cdots+\lambda_{n} e_{n}$, where $\lambda_{1}, \lambda_{2}, \ldots, \lambda_{n}$ are the eigenvalues of $x$ and $\left\{e_{1}, e_{2}, \ldots, e_{n}\right\}$ is a Jordan frame in $V$. Suppose, if possible, $x \nsupseteq 0$; we may assume without loss of generality that $\lambda_{1}<0$. Then, $x \circ e_{1}=\lambda_{1} e_{1}$ and

$$
\begin{aligned}
0 & \leq\left\langle x+y, e_{1}\right\rangle=\left\langle x, e_{1}\right\rangle+\left\langle y, e_{1}\right\rangle=\lambda_{1}\left\|e_{1}\right\|^{2}+\frac{1}{\lambda_{1}}\left\langle y, x \circ e_{1}\right\rangle \\
& =\lambda_{1}\left\|e_{1}\right\|^{2}+\frac{1}{\lambda_{1}}\left\langle x \circ y, e_{1}\right\rangle=\lambda_{1}\left\|e_{1}\right\|^{2}+\frac{1}{\lambda_{1}}\left\langle w, e_{1}\right\rangle<0,
\end{aligned}
$$

as $\left\langle w, e_{1}\right\rangle \geq 0$. This contradiction proves that all eigenvalues of $x$ are nonnegative; so $x \geq 0$. Similarly, $y \geq 0$. Thus we have $(i v)$.

Item $(i v)$ in the above proposition will allow us to formulate a wHLCP as a system of equations. In fact, $(x, y)$ is a solution of $\operatorname{wHLCP}(A, B, w, q)$ (4) if and only if it is a solution of the system

$$
\begin{gathered}
x+y-\sqrt{x^{2}+y^{2}+2 w}=0, \\
A x+B y-q=0 .
\end{gathered}
$$

Our next key result will be used to show that the min and the FischerBurmeister maps are 'homotopic'. This will allow us to replace the FischerBurmeister map by the 'simpler' min map in our main solution analysis.

Proposition 2 Let $x, y \in V$ and $0 \leq t \leq 1$. Then,

$$
t\left[x+y-\sqrt{x^{2}+y^{2}}\right]+(1-t) x \sqcap y=0 \Longleftrightarrow x \sqcap y=0 .
$$

Proof. In view of Items (iii) and (iv) in the previous proposition, we prove only the 'if' part. We also assume without loss of generality, $0<t<1$. Let $u:=t\left[x+y-\sqrt{x^{2}+y^{2}}\right]$. From Item $(i)$ of the previous proposition,

$$
[(1-t) x+u] \sqcap[(1-t) y+u]=0 .
$$


This implies that $(1-t) x+u \geq 0$ and $(1-t) y+u \geq 0$. Now, $(1-t) x+u \geq 0$ implies that $(1-t) x+t\left[x+y-\sqrt{x^{2}+y^{2}}\right] \geq 0$, that is, $x+t y \geq t \sqrt{x^{2}+y^{2}}$. As $\sqrt{x^{2}+y^{2}} \geq \sqrt{y^{2}}=|y|$ (which is a consequence of the so-called LöwnerHeinz inequality, see [7], Proposition 8), we see that

$$
x \geq t|y|-t y \geq 0
$$

Hence $x \geq 0$. Similarly, $y \geq 0$. It follows that $\langle x, y\rangle \geq 0$. We now show that $\langle x, y\rangle \leq 0$ to conclude that $\langle x, y\rangle=0$.

We first note that $x \sqcap y=(x+y-|x-y|) / 2$. Let

$$
p:=\frac{t}{1-t}\left[x+y-\sqrt{x^{2}+y^{2}}\right]
$$

so that $x+y-\sqrt{x^{2}+y^{2}}=\alpha p$, where $\alpha:=\frac{1-t}{t}$. Then, $\sqrt{x^{2}+y^{2}}=(x+y)-\alpha p$. Squaring both sides and simplifying, we get

$$
p \circ(x+y)=\frac{1}{2 \alpha}\left[2 x \circ y+\alpha^{2} p^{2}\right] .
$$

As $t\left[x+y-\sqrt{x^{2}+y^{2}}\right]+(1-t) x \sqcap y=0$, we have $p+x \sqcap y=0$, that is,

$$
2 p+(x+y)=|x-y| \text {. }
$$

Squaring both sides, noting $|x-y|^{2}=(x-y)^{2}$, and simplifying, we get

$$
4 p^{2}+2 x \circ y+4 p \circ(x+y)=-2 x \circ y \text {. }
$$

We replace $4 p \circ(x+y)$ by using (5) to get an expression of the form

$$
\beta x \circ y+\gamma p^{2}=0
$$

where numbers $\beta$ and $\gamma$ are positive. This yields $x \circ y \leq 0$ and $\langle x, y\rangle=$ $\langle x \circ y, e\rangle \leq 0$. Finally, since $\langle x, y\rangle \geq 0$, we have $\langle x, y\rangle=0$. Thus we have shown that $x, y \geq 0$ and $\langle x, y\rangle=0$. Hence, $x \sqcap y=0$.

We end this subsection by quoting a well-known determinantal formula.

Proposition 3 [12] For $A, B, X, Y \in \mathbb{R}^{n \times n}$, with $X, Y$ commuting, the following formula holds:

$$
\operatorname{det}\left[\begin{array}{cc}
A & -B \\
X & Y
\end{array}\right]=\operatorname{det}(A Y+B X)
$$

A similar statement can be made about linear transformations. 


\subsection{Degree theory}

We employ degree theoretic arguments to prove our existence results. All necessary results concerning degree theory are given in 4]. (specifically Proposition 2.1.3); see also, [10, 11. Here is a brief summary. Suppose $\Omega$ is a bounded open set in $\mathbb{R}^{n}, g: \bar{\Omega} \rightarrow \mathbb{R}^{n}$ is continuous and $p \notin g(\partial \Omega)$, where $\bar{\Omega}$ and $\partial \Omega$ denote, respectively, the closure and boundary of $\Omega$. Then the degree of $g$ over $\Omega$ with respect to $p$ is defined; it is an integer and will be denoted by $\operatorname{deg}(g, \Omega, p)$. When this degree is nonzero, the equation $g(x)=p$ has a solution in $\Omega$. Suppose $g(x)=p$ has a unique solution, say, $x^{*}$ in $\Omega$. Then $\operatorname{deg}\left(g, \Omega^{\prime}, p\right)$, which equals $\operatorname{deg}\left(g, \Omega^{\prime}, g\left(x^{*}\right)\right)$, is constant over all bounded open sets $\Omega^{\prime}$ containing $x^{*}$ and contained in $\Omega$. This common degree is called the (topological) index of $g$ at $x^{*}$; it will be denoted by ind $\left(g, x^{*}\right)$. In particular, if $g: \mathbb{R}^{n} \rightarrow \mathbb{R}^{n}$ is a continuous map such that $g(x)=0 \Leftrightarrow x=0$, then for any bounded open set containing 0 , we have

$$
\operatorname{ind}(g, 0)=\operatorname{deg}(g, \Omega, 0)
$$

moreover, when $g$ is the identity $\operatorname{map}, \operatorname{ind}(g, 0)=1$.

Let $H(x, t): \mathbb{R}^{n} \times[0,1] \rightarrow \mathbb{R}^{n}$ be continuous (in which case, we say that $H$ is a homotopy). Suppose that for some bounded open set $\Omega$ in $\mathbb{R}^{n}, 0 \notin H(\partial \Omega, t)$ for all $t \in[0,1]$. Then, the homotopy invariance property of degree says that $\operatorname{deg}(H(\cdot, t), \Omega, 0)$ is independent of $t$. In particular, if the zero set

$$
\{x: H(x, t)=0 \text { for some } t \in[0,1]\}
$$

is bounded, then for any bounded open set $\Omega$ in $\mathbb{R}^{n}$ containing this zero set, we have

$$
\operatorname{deg}(H(\cdot, 1), \Omega, 0)=\operatorname{deg}(H(\cdot, 0), \Omega, 0) .
$$

Note: All degree theory concepts and results are also valid over any finite dimensional real Hilbert space (such as $V$ or $V \times V$ ) instead of $\mathbb{R}^{n}$.

\subsection{A normalization argument}

To show that the zero set of a map or a system of equations is bounded, we frequently employ the so-called normalization argument. Here, a certain sequence of vectors (with their norms going to infinity) is normalized to yield a unit vector that violates a given criteria. We illustrate this in the following result, which will be used later.

Proposition 4 Let $A$ and $B$ two linear transformations on $V$ and $p \in V$. Suppose that

$$
[x \sqcap y=0, A x+B y=0] \Rightarrow(x, y)=(0,0) .
$$


Then, the set

$$
\{(x, y): x \sqcap y=0, A x+B y-t p=0 \text { for some } t \in[0,1]\}
$$

is bounded.

Proof. Suppose the above set is unbounded. Let $z_{k}:=\left(x_{k}, y_{k}\right), t_{k} \in[0,1]$ with $\left\|z_{k}\right\| \rightarrow \infty$, and $x_{k} \sqcap y_{k}=0$, and $A x_{k}+B y_{k}-t_{k} p=0$ for all $k=1,2, \ldots$ We divide each of the above equations by $\left\|z_{k}\right\|$ (so as to create normalized vectors $\left.\frac{z_{k}}{\left\|z_{k}\right\|}\right)$. We let $k \rightarrow \infty$ and suppose without loss of generality, $x_{0}:=\lim \frac{x_{k}}{\left\|z_{k}\right\|}$ and $y_{0}:=\lim \frac{y_{k}}{\left\|z_{k}\right\|}$. Then, $x_{0} \sqcap y_{0}=0$, and $A x_{0}+B y_{0}=0$. However, from $\left\|z_{k}\right\|^{2}=\left\|x_{k}\right\|^{2}+\left\|y_{k}\right\|^{2}$, we get $\left\|x_{0}\right\|^{2}+\left\|y_{0}\right\|^{2}=1$, which contradicts our assumption. The stated conclusion follows.

\section{The degree of an $R_{0}$-pair}

In the setting of $V=\mathbb{R}^{n}$, the concepts of LCP-degree of a matrix and HLCPdegree of a pair of matrices are useful in describing the existence and stability of solutions, see [6] and [15]. In what follows, we extend these to Euclidean Jordan algebras. Consider linear transformations $M, A$, and $B$ on (a general algebra) $V$ and recall that

$\operatorname{HLCP}(A, B, q):=\operatorname{wHLCP}(A, B, 0, q) \quad$ and $\operatorname{LCP}(M, q):=\operatorname{HLCP}(I,-M, q)$.

In view of Item (iii) in Proposition 1, $\operatorname{HLCP}(A, B, q)$ is equivalent to finding $(x, y) \in V \times V$ such that

$$
\begin{gathered}
x \sqcap y=0, \\
A x+B y=q,
\end{gathered}
$$

and $\operatorname{LCP}(M, q)$ is equivalent to finding an $x \in V$ such that

$$
x \sqcap(M x+q)=0 .
$$

We say that $M$ has the $\mathbf{R}_{0}$ property on $V$ if zero is the only solution of $\operatorname{LCP}(M, 0)$, or equivalently,

$$
x \sqcap M x=0 \Leftrightarrow x=0 .
$$

When this condition holds, for any bounded open set $\Omega$ in $V$ that contains zero, $\operatorname{deg}(\theta, \Omega, 0)$ is well defined, where

$$
\theta(x):=x \sqcap M x .
$$

This common value - which is ind $(\theta, 0)$ - defines the LCP-degree of $M$, denoted by $\operatorname{deg}(M)$. We now extend this concept to a pair of transformations. 
Definition 1 The pair $\{A, B\}$ is said to be an $\mathbf{R}_{0}$-pair if zero is the only solution of $\operatorname{HLCP}(A, B, 0)$. This means that

$$
\Theta(z)=0 \Leftrightarrow z=0
$$

where

$$
\Theta(z):=\left[\begin{array}{c}
x \sqcap y \\
A x+B y
\end{array}\right]
$$

with $z=(x, y)$. When this condition holds, $\operatorname{ind}(\Theta, 0)$ is well defined. (Note that this equals $\operatorname{deg}(\Theta, \Omega, 0)$ for any bounded open set $\Omega$ in $V \times V$ that contains zero.) We define the HLCP-degree of the pair $\{A, B\}$ by

$$
\operatorname{deg}(A, B):=\operatorname{ind}(\Theta, 0) .
$$

Our first result extends Corollary 5.2.6 in [15] from $\mathbb{R}^{n}$ to a general Euclidean Jordan algebra.

Proposition $\mathbf{5}$ Let $M$ be a linear transformation on $V$ with the $\mathbf{R}_{0}$ property. Then $\{I,-M\}$ is an $\mathbf{R}_{0}$-pair and

$$
\operatorname{deg}(I,-M)=\operatorname{deg}(M) .
$$

Proof. It is easy to see that $\{I,-M\}$ is an $\mathbf{R}_{0}$-pair. Now define the map

$$
\Theta(z, t):=\left[\begin{array}{c}
y \sqcap[t x+(1-t) M y] \\
x-t M y
\end{array}\right],
$$

where $z=(x, y)$ and $t \in[0,1]$. Then,

$$
\Theta(z, 0)=\left[\begin{array}{c}
\theta(y) \\
x
\end{array}\right] \text { and } \Theta(z, 1)=\Theta(z)
$$

where $\theta(y)=y \sqcap M y$. Moreover, since $M$ has the $\mathbf{R}_{0}$ property (so that $y \sqcap M y=0 \Rightarrow y=0)$, it is easy to verify that $\Theta(z, t)=(0,0) \in V \times V$ if and only if $z=0$. This means that the zero set of $\Theta(z, t)$ (as $t$ varies over $[0,1]$ ) is just $\{(0,0)\}$. Thus, for arbitrary bounded open sets $\Omega_{1}$ and $\Omega_{2}$ both containing zero in $V$, letting $\Omega=\Omega_{1} \times \Omega_{2}$, we have, by the homotopy invariance of degree and the Cartesian product formula (see 4], Proposition 2.1.3(h)),

$$
\begin{aligned}
\operatorname{deg}(I,-M) & =\operatorname{deg}(\Theta(\cdot, 1), \Omega, 0)=\operatorname{deg}(\Theta(\cdot, 0), \Omega, 0) \\
& =\operatorname{deg}\left(\theta, \Omega_{1}, 0\right) \operatorname{deg}\left(I, \Omega_{2}, 0\right)=\operatorname{ind}(\theta, 0)=\operatorname{deg}(M),
\end{aligned}
$$

where $I$ denotes the identity transformation.

In the standard LCP theory, R-matrices [2] form an important subclass of matrices for which LCP-degree is nonzero. (We note that there are other matrices, such as $\mathbf{N}$-matrices of first category satisfying this property [6.) Recall that $M$ is an $\mathbf{R}$-matrix (in the standard LCP setting) if there is some $d>0$ in $\mathbb{R}^{n}$ such that zero is the only vector that solves the problems $\operatorname{LCP}(M, 0)$ and $\operatorname{LCP}(M, d)$. We now consider a generalization. 
Definition 2 Let $A$ and $B$ be two linear transformations on $V$. We say that $\{A, B\}$ is an $\mathbf{R}$-pair if it is an $\mathbf{R}_{0}$-pair and there exists $p \in V$ such that

(a) $\operatorname{HLCP}(A, B, p)$ has a unique solution, say, $(\bar{x}, \bar{y})$,

(b) $\bar{x}+\bar{y}>0$, and

(c) The derivative of $G$ at $(\bar{x}, \bar{y})$ is nonsingular, where

$$
G(z):=\left[\begin{array}{c}
x \sqcap y \\
A x+B y-p
\end{array}\right]
$$

with $z=(x, y)$.

Note that condition $(c)$ above is equivalent to: The derivative of $\Theta$ (as given in the Definition of $\mathbf{R}_{0}$-pair) at $(\bar{x}, \bar{y})$ is nonsingular.

We elaborate on the $\mathbf{R}$-pair property and give some examples. Suppose $\{A, B\}$ is an $\mathbf{R}$-pair. As $\bar{x} \sqcap \bar{y}=0$ and $\bar{x}+\bar{y}>0, \bar{x}$ and $\bar{y}$ operator commute (see Proposition 1) and so, with respect to some Jordan frame $\left\{e_{1}, e_{2}, \ldots, e_{n}\right\}$, we can write

$$
\bar{x}=\sum_{1}^{k} \bar{x}_{i} e_{i} \quad \text { and } \quad \bar{y}=\sum_{k+1}^{n} \bar{y}_{j} e_{j},
$$

where $1 \leq k \leq n$ and $\bar{x}_{i}, \bar{y}_{j}>0$ for all $i$ and $j$. Let $\bar{z}:=(\bar{x}, \bar{y})$,

$$
\alpha:=\{1,2, \ldots, k\} \quad \text { and } \quad \beta=\{k+1, \ldots, n\} .
$$

(Note that one of these sets may be empty.) Thus, the element

$$
\bar{x}-\bar{y}=\sum_{1}^{k} \bar{x}_{i} e_{i}-\sum_{k+1}^{n} \bar{y}_{i} e_{i}
$$

is invertible (which means that all the eigenvalues are nonzero). In view of Lemma 19 in [7, the map $G$ defined in condition $(c)$ is Fréchet differentiable. Let

$$
\Phi(x, y):=x \sqcap y=x-\Pi_{V_{+}}(x-y),
$$

where $\Pi_{V_{+}}$denotes the projection operator onto $V_{+}$. Then, the partial derivative of $\Phi$ with respect to $x$ at $\bar{z}=(\bar{x}, \bar{y})$ is given by

$$
\Phi_{x}^{\prime}(\bar{z})=I_{x}-\Pi_{V_{+}}^{\prime}(\bar{x}-\bar{y}) \circ I_{x}
$$

where $I_{x}$ denotes the identity transformation and ' $\mathrm{O}$ ' denotes the composition. Now, we use the formula for the derivative of $\Pi_{V_{+}}$given in Lemma 19 of 7 . (Although this formula is stated in the setting of a simple Euclidean Jordan algebra, by writing a general Euclidean Jordan algebra as a product of simple ones, we can show that that the formula is valid in any Euclidean Jordan 
algebra.) Then, for any $h \in V$ with Peirce decomposition $h=\sum_{1}^{n} h_{i} e_{i}+$ $\sum_{i<j} h_{i j}$, we have

$$
\Phi_{x}^{\prime}(\bar{z}) h=h-\left(\sum_{1}^{k} h_{i} e_{i}+\sum_{i, j \in \alpha ; i<j} h_{i j}+\sum_{i \in \alpha, j \in \beta} \frac{\bar{x}_{i}}{\bar{x}_{i}+\bar{y}_{j}} h_{i j}\right) .
$$

Simplification leads to

$$
\Phi_{x}^{\prime}(\bar{z}) h=\sum_{k+1}^{n} h_{i} e_{i}+\sum_{i, j \in \beta ; i<j} h_{i j}+\sum_{i \in \alpha, j \in \beta} \frac{\bar{y}_{j}}{\bar{x}_{i}+\bar{y}_{j}} h_{i j} .
$$

Similarly, using $\Phi(x, y):=y \sqcap x=y-\Pi_{V_{+}}(y-x)$, we see that the $y$-derivative of $\Phi(x, y)$ at $(\bar{x}, \bar{y})$ is given by

$$
\Phi_{y}^{\prime}(\bar{z}) h=\sum_{1}^{k} h_{i} e_{i}+\sum_{i, j \in \alpha ; i<j} h_{i j}+\sum_{i \in \alpha, j \in \beta} \frac{\bar{x}_{i}}{\bar{x}_{i}+\bar{y}_{j}} h_{i j} .
$$

Thus, the nonsingularity of $G$ at $(\bar{x}, \bar{y})$ could be expressed by the implication

$$
\left[\Phi_{x}^{\prime}(\bar{z}) u+\Phi_{y}^{\prime}(\bar{z}) v=0 \text { and } A u+B v=0\right] \Rightarrow(u, v)=(0,0) .
$$

Upon simplification, the above condition could be written as

$$
\left.\begin{array}{c}
u_{i}=0(i \in \beta), v_{j}=0(j \in \alpha) \\
u_{i j}=0(i, j \in \beta ; i<j), v_{i j}=0(i, j \in \alpha ; i<j) \\
u_{i j}=-\frac{\bar{x}_{i}}{\bar{y}_{j}} v_{i j}(i \in \alpha, j \in \beta) \\
A u+B v=0
\end{array}\right\} \Rightarrow(u, v)=(0,0) .
$$

We now show that in some standard settings, condition $(c)$ in Definition 2 is a consequence of conditions $(a)$ and $(b)$.

Example 1 Let $V=\mathbb{R}^{n}$. Then the standard coordinate vectors form the only Jordan frame and for any element $h \in \mathbb{R}^{n}, h_{i j}=0$ for all $i<j$. Thus, in order to verify the implication (8), we let

$$
u=\left(u_{1}, u_{2}, \ldots, u_{k}, 0,0, \ldots, 0\right)^{T} \text { and } v=\left(0,0, \ldots, 0, v_{k+1}, \ldots, v_{n}\right)^{T}
$$

and suppose that $(u, v) \neq(0,0)$. As $\bar{x}=\left(\bar{x}_{1}, \bar{x}_{2}, \ldots, \bar{x}_{k}, 0,0, \ldots, 0\right)^{T}$ and $\bar{y}=\left(0,0, \ldots, 0, \bar{y}_{k+1}, \ldots \bar{y}_{n}\right)^{T}$, where $\bar{x}_{i}$ and $\bar{y}_{j}$ are positive, we see that for all small $\varepsilon>0$, the pair $(\bar{x}+\varepsilon u, \bar{y}+\varepsilon v)$ is a solution of $\operatorname{HLCP}(A, B, p)$. This contradicts the uniqueness assumption $(a)$. Hence condition $(c)$ is superfluous in this setting.

Example 2 Let $M: V \rightarrow V$ be a linear transformation that has the $\mathbf{R}$ property with respect to $V_{+}$. This means that for some $d>0$ in $V$, zero is the only solution of the linear complementarity problems $\operatorname{LCP}(M, 0)$ and $\operatorname{LCP}(M, d)$. We claim that $\{I,-M\}$ is an $\mathbf{R}$-pair. It is easy to see that the 
problem $\operatorname{HLCP}(I,-M, 0)$ has $(0,0)$ as the only solution which means that $\{I,-M\}$ is an $\mathbf{R}_{0}$-pair. Also, $\operatorname{HLCP}(I,-M, d)$ has $(d, 0)$ as the only solution. This means that with $(\bar{x}, \bar{y})=(d, 0)$, conditions $(a)$ and $(b)$ in the above definition are satisfied. We show that condition $(c)$ holds. If $z=(x, y)$ is close to $(d, 0)$, then $x-y$ is close to $d-0$; hence for all such $(x, y), \Pi_{V_{+}}(x-y)=x-y$ and $x \sqcap y=x-(x-y)=y$. Thus, when $z$ is close to $(d, 0)$, we have

$$
G(z):=\left[\begin{array}{c}
y \\
I x-M y-p
\end{array}\right] \text { and } \quad G^{\prime}(\bar{z}):=\left[\begin{array}{cc}
0 & I \\
I & -M
\end{array}\right] .
$$

In view of Proposition $3, G^{\prime}(\bar{z})$ is nonsingular. Thus, we have verified condition (c). Hence $\{I,-M\}$ is an $\mathbf{R}$-pair.

Proposition 6 Suppose $\{A, B\}$ is an $\mathbf{R}$-pair. Then, $\operatorname{deg}(A, B)$ is nonzero.

Proof. The pair $\{A, B\}$ satisfies conditions in Definition 2 Let

$$
G(z, t):=\left[\begin{array}{c}
x \sqcap y \\
A x+B y-t p
\end{array}\right]
$$

where $z=(x, y)$ and $t \in[0,1]$. Then,

$$
G(z, 1)=\left[\begin{array}{c}
x \sqcap y \\
A x+B y-p
\end{array}\right] \quad \text { and } \quad G(z, 0)=\left[\begin{array}{c}
x \sqcap y \\
A x+B y
\end{array}\right] .
$$

As $\{A, B\}$ is an $\mathbf{R}_{0}$-pair, by a normalization argument (see Proposition 4 ), we see that the zero sets of $G(z, t)$ as $t$ varies are uniformly bounded. Suppose $\Omega$ is a bounded open set in $V \times V$ that contains all these zero sets. Note that $G(z, 1)$ vanishes only at $(\bar{x}, \bar{y}) \in \Omega$ and its derivative at this point is nonsingular. Thus,

$$
\operatorname{deg}(A, B)=\operatorname{deg}(G(\cdot, 0), \Omega, 0)=\operatorname{deg}(G(\cdot, 1), \Omega, 0)=\operatorname{sgn} \operatorname{det} G^{\prime}(\bar{z}, 1) \neq 0 .
$$

This proves that $\operatorname{deg}(A, B)$ is nonzero.

\section{The main existence result}

We now discuss the solvability of $\operatorname{wHLCP}(A, B, w, q)$. We recall that $(x, y)$ is a solution of $\operatorname{wHLCP}(A, B, w, q)$ if and only if it is a solution of the system

$$
\begin{gathered}
x+y-\sqrt{x^{2}+y^{2}+2 w}=0, \\
A x+B y-q=0 .
\end{gathered}
$$

We show that this system has a solution under a nonzero degree condition.

Theorem 1 Let $\{A, B\}$ be an $\mathbf{R}_{0}$-pair with $\operatorname{deg}(A, B)$ nonzero. Then for any $(w, q) \in V_{+} \times V, \operatorname{wHLCP}(A, B,, w, q)$ has a nonempty compact solution set. 
Proof We fix $(w, q) \in V_{+} \times V$. With $z=(x, y) \in V \times V$ and $t \in[0,1]$, we define the following maps:

$$
\begin{gathered}
F(z, t):=\left[\begin{array}{c}
x+y-\sqrt{x^{2}+y^{2}+2 t w} \\
A x+B y-t q
\end{array}\right], \\
H(z, t):=\left[\begin{array}{c}
t\left[x+y-\sqrt{x^{2}+y^{2}}\right]+(1-t) x \sqcap y \\
A x+B y
\end{array}\right] .
\end{gathered}
$$

We show below that there is some bounded open set $\Omega$ in $V \times V$ which contains all the zeros (in $z$ ) of $F$ and $H$ (as $t$ varies over $[0,1]$ ). Then, over $\Omega, F$ is a homotopy connecting $F(z, 1)$ and $F(z, 0) ; H$ is a homotopy connecting $H(z, 1)$ $(=F(z, 0))$ and $H(z, 0)$. Using the homotopy invariance property of degree, we see that

$$
\operatorname{deg}(F(\cdot, 1), \Omega, 0)=\operatorname{deg}(H(\cdot, 0), \Omega, 0) \neq 0
$$

This shows that the equation $F(z, 1)=0$ has a nonempty bounded solution set. Let

To justify these, we proceed as follows.

$$
Z:=\{z: F(z, t)=0 \text { for some } t \in[0,1]\} .
$$

We show by a normalization argument that $Z$ is bounded. Suppose, if possible, $Z$ is unbounded. Let $z_{k}:=\left(x_{k}, y_{k}\right), t_{k} \in[0,1]$ with $\left\|z_{k}\right\| \rightarrow \infty$, and $F\left(z_{k}, t_{k}\right)=$ 0 for all $k=1,2, \ldots$ Let $k \rightarrow \infty$ and without loss of generality, $x_{0}:=\lim \frac{x_{k}}{\left\|z_{k}\right\|}$ and $y_{0}:=\lim \frac{y_{k}}{\| z_{k}}$. We note that $\left\|x_{0}\right\|^{2}+\left\|y_{0}\right\|^{2}=1$. Dividing each component of $F\left(z_{k}, t_{k}\right)$ by $\left\|z_{k}\right\|$ and letting $k \rightarrow \infty$, we get

$$
\begin{gathered}
x_{0}+y_{0}-\sqrt{x_{0}^{2}+y_{0}^{2}}=0, \\
A x_{0}+B y_{0}=0 .
\end{gathered}
$$

By Proposition $1,\left(x_{0}, y_{0}\right)$ becomes a nonzero solution of $\operatorname{HLCP}(A, B, 0)$ contradicting the $\mathbf{R}_{0}$ property of $\{A, B\}$. Hence, $Z$ is bounded. Next, in view of Proposition 2 and the $\mathbf{R}_{0}$ property of $\{A, B\}$,

$$
\{z: H(z, t)=0 \text { for some } t \in[0,1]\}=\{(0,0)\} .
$$

Let $\Omega$ be a bounded open set in $V \times V$ that contains the zero sets of $F$ and $H$. Then, by the homotopy invariance property of the degree,

$\operatorname{deg}(F(\cdot, 1), \Omega, 0)=\operatorname{deg}(F(\cdot, 0), \Omega, 0)=\operatorname{deg}(H(\cdot, 1), \Omega, 0)=\operatorname{deg}(H(\cdot, 0), \Omega, 0)$

and

$$
\operatorname{deg}(H(\cdot, 0), \Omega, 0)=\operatorname{deg}(\Theta, \Omega, 0)=\operatorname{ind}(\Theta, 0)=\operatorname{deg}(A, B)
$$


As the last quantity, by assumption, is nonzero, we conclude that

$$
\operatorname{deg}(F(\cdot, 1), \Omega, 0) \neq 0 .
$$

This means that the equation $F(z, 1)=0$ has a zero in $\Omega$ proving the existence of a solution of $\operatorname{wHLCP}(A, B, w, q)$. As all zeros of $F(\cdot, 1)$ are in the bounded set $\Omega$ and the solution set of $\operatorname{wHLCP}(A, B, w, q)$ is clearly closed, we see nonemptyness and compactness of this solution set. This completes the proof.

Motivated by interior point methods, we consider the case $w>0$. First, we make a simple observation:

$$
[x \geq 0, y \geq 0 \text {, and } x \circ y>0] \Longrightarrow x>0 \text { and } y>0 .
$$

This follows from Item (iv), Lemma 2.6 in [18. Here is a short/different proof. Let $x \geq 0, y \geq 0$ and $x \circ y=w>0$. Suppose $x \ngtr 0$ so that zero is an eigenvalue of $x$. This means that there is a primitive idempotent $e_{1}$ (which belongs to the Jordan frame that appears in the spectral decomposition of $x$ ) such that $x \circ e_{1}=0$. But then, $0<\left\langle w, e_{1}\right\rangle=\left\langle x \circ y, e_{1}\right\rangle=\left\langle y, x \circ e_{1}\right\rangle=0$, is a contradiction. Hence, $x>0$ and, similarly, $y>0$.

Corollary 1 Let $\{A, B\}$ be an $\mathbf{R}_{0}$-pair with $\operatorname{deg}(A, B)$ nonzero. Suppose $w>$ 0 . Then for any $q \in V$, the following 'interior point system' has a nonempty compact solution set:

$$
x>0, y>0, x \circ y=w, \text { and } A x+B y=q .
$$

We now specialize the above two results for a single linear transformation.

Corollary 2 Let $M$ be a linear transformation on $V$. Suppose that $M$ has the $\mathbf{R}_{0}$ property and $\operatorname{deg}(M)$ is nonzero. Then, for all $(w, q) \in V_{+} \times V$, the weighted linear complementarity problem

$$
x \geq 0, y \geq 0, x \circ y=w, \text { and } y=M x+q
$$

has a nonempty compact solution set. In particular, when $w>0$, for any $q \in V$, the 'interior point system'

$$
x>0, y>0, x \circ y=w, \text { and } y=M x+q
$$

has a nonempty compact solution set.

Remarks. In the standard LCP literature, the solvability and uniqueness issues of interior point systems are usually addressed for special types of $\mathbf{P}_{0^{-}}$ matrices (e.g., $\mathbf{P}_{*}$-matrices), see 9, Lemma 4.3 and Theorem 4.4. In this regard, the above result appears to be new even in the case of $V=\mathbb{R}^{n}$, as it holds for numerous types of non $\mathbf{P}_{0}$-matrices such as strictly copositive matrices, $\mathbf{R}$-matrices, and $\mathbf{N}$-matrices of first category [2]. 


\section{The solution set behavior}

Fixing the pair $\{A, B\}$, we let $\operatorname{SOL}(w, q)$ denote the solution set of ${ }_{w} \operatorname{HLCP}(A, B, w, q)$. The following result describes the behavior of the map $(w, q) \mapsto \operatorname{SOL}(w, q)$.

Theorem 2 Suppose $\{A, B\}$ is an $\mathbf{R}_{0}$-pair with $\operatorname{deg}(A, B)$ nonzero. Then, the following statements hold:

(a) The solution map $(w, q) \mapsto \operatorname{SOL}(w, q)$ from $V_{+} \times V$ to $V_{+} \times V_{+}$is upper semicontinuous.

(b) Let $w_{k} \geq 0$ for all $k=1,2, \ldots$, and $w_{k} \rightarrow w$. Suppose $\left(x_{k}, y_{k}\right) \in \operatorname{SOL}\left(w_{k}, q\right)$ for all $k$. Then, the sequence $\left\{\left(x_{k}, y_{k}\right)\right\}$ is bounded and any accumulation point of this sequence solves $\mathrm{wHLCP}(A, B, w, q)$.

(c) Let $w>0, t_{k} \downarrow 0$, and $\left(x_{k}, y_{k}\right) \in \operatorname{SOL}\left(t_{k} w, q\right)$ for all $k$. Then, $x_{k}>0$ and $y_{k}>0$ for all $k$, the sequence $\left\{\left(x_{k}, y_{k}\right)\right\}$ is bounded, and any accumulation point of this sequence solves $\operatorname{HLCP}(A, B, q)$.

Proof. (a) We fix $\left(w^{*}, q^{*}\right) \in V_{+} \times V$ and let $\Omega$ be any open set in $V \times V$ containing $\operatorname{SOL}\left(w^{*}, q^{*}\right)$. We show that for all $(w, q)$ near $\left(w^{*}, q^{*}\right), \operatorname{SOL}(w, q)$ is contained in $\Omega$. Assuming the contrary, suppose there is a sequence $\left\{\left(w_{k}, q_{k}\right)\right\}$ converging to $\left(w^{*}, q^{*}\right)$ such that some solution $\left(x_{k}, y_{k}\right)$ in $\operatorname{SOL}\left(w_{k}, q_{k}\right)$ belongs to $\Omega^{c}$ (the complement of $\Omega$ ). The sequence $\left\{\left(x_{k}, y_{k}\right)\right\}$ has to be bounded; else, a normalization argument (such as the one used in the previous theorem) produces a nonzero solution of $\operatorname{HLCP}(A, B, 0)$ contradicting the $\mathbf{R}_{0}$ property of the pair $\{A, B\}$. Now, a subsequential limit of the sequence belongs to $\operatorname{SOL}\left(w^{*}, q^{*}\right)$ and at the same time is in $\Omega^{c}$ (as this set is closed). This contradiction proves the upper semicontinuity property of the solution set.

(b) Under the stated assumptions, $\left(w_{k}, q\right) \rightarrow(w, q)$. A normalization argument shows that the sequence $\left\{\left(x_{k}, y_{k}\right)\right\}$ is bounded. Any subsequential limit of this sequence, clearly, belongs to $\operatorname{SOL}(w, q)$, that is, $\operatorname{solves} \operatorname{wHLCP}(A, B, w, q)$.

(c) That $x_{k}>0$ and $y_{k}>0$ for all $k$ follows from Corollary 1 , For the remaining statements, we specialize $(b)$ with $w_{k}:=t_{k} w$.

\section{P-pairs over $V$}

A linear transformation $M$ on $V$ is said to be a $\mathbf{P}$-transformation [7] if

$$
\left.\begin{array}{r}
x \text { and } M x \text { operator commute } \\
x \circ M x \leq 0
\end{array}\right\} \Rightarrow x=0 .
$$

$\mathbf{P}$-transformations are generalizations of $\mathbf{P}$-matrices. An important example of P-transformations appears in dynamical systems: the Lyapunov transformation $X \mapsto A X+X A^{T}$ on the Euclidean Jordan algebra of $n \times n$ real symmetric matrices is a $\mathbf{P}$-transformation if and only if the (real square) $A$ is positive stable. See [7] for properties of $\mathbf{P}$-transformations and further examples. We now extend this notion to a pair of transformations. 
Definition 3 A pair of linear transformations $\{A, B\}$ is said to be a $\mathbf{P}$-pair over $V$ if

$$
\left.\begin{array}{r}
x \text { and } y \text { operator commute } \\
x \circ y \leq 0 \\
A x+B y=0
\end{array}\right\} \Rightarrow(x, y)=(0,0) .
$$

Below, we collect some properties of such pairs.

Proposition 7 Suppose $\{A, B\}$ is a $\mathbf{P}$-pair. Then, the following statements hold.

(a) $A$ and $B$ are invertible.

(b) $-B^{-1} A$ and $-A^{-1} B$ are $\mathbf{P}$-transformations.

(c) $\{A, B\}$ is an $\mathbf{R}$-pair.

(d) For all $(w, q) \in V_{+} \times V, \operatorname{wHLCP}(A, B, w, q)$ has a nonempty compact solution set.

Proof. (a) If $A x=0$ for some $x$, then

$[x$ and 0 operator commute, $x \circ 0=0, A x+B 0=0] \Rightarrow(x, 0)=(0,0)$.

This shows that $A$ is invertible. Similarly $B$ is invertible.

(b) Let $M:=-B^{-1} A$. If $x$ and $M x$ operator commute and $x \circ M x \leq 0$, then, with $y:=M x=-B^{-1} A x$, we see that: $x$ and $y$ operator commute, $x \circ y \leq 0$, and $A x+B y=0$. Hence $(x, y)=(0,0)$ and so $x=0$. Thus, $-B^{-1} A$ is a $\mathbf{P}$-transformation. Similarly, $-A^{-1} B$ is also a $\mathbf{P}$-transformation.

(c) We now show that $\{A, B\}$ is an $\mathbf{R}$-pair. First, $\{A, B\}$ is an $\mathbf{R}_{0}$-pair: When $x \sqcap y=0$ and $A x+B y=0$, by Proposition 2, $x$ and $y$ operator commute, $x \circ y=0$, and $A x+B y=0$; so, $(x, y)=(0,0)$ by the definition of a $\mathbf{P}$-pair.

Now let $p:=B e$, where $e$ denotes the unit element in $V$. Clearly, $(0, e)$ is a solution of $\operatorname{HLCP}(A, B, p)$. We show that this is the only solution. Let $(x, y)$ be any solution of $\operatorname{HLCP}(A, B, p)$ so that $x$ and $y$ operator commute, $x, y \geq 0$, and $x \circ y=0$. This implies that $x$ and $y-e$ operator commute and $x \circ(y-e)=-x \leq 0$. Since we also have $A x+B(y-e)=0$, by the definition of $\mathbf{P}$-pair, $(x, y-e)=(0,0)$. This implies that $(x, y)=(0, e)$. Since $0+e=e>0$, we see that conditions $(a)$ and $(b)$ in Definition 2 hold. We now verify condition $(c)$ in that definition. If $(x, y)$ is close to $(0, e)$, then $x-y$ is close to $-e$ and so $\Pi_{V_{+}}(x-y)=0$. In this case, $x \sqcap y=x-\Pi_{V_{+}}(x-y)=x$. Hence, for all $z=(x, y)$ near $\bar{z}:=(0, e)$,

$$
G(z):=\left[\begin{array}{c}
x \\
A x+B y-B e
\end{array}\right] \text { and } G^{\prime}(\bar{z}):=\left[\begin{array}{cc}
I & 0 \\
A & B
\end{array}\right] .
$$

In view of Proposition 3 and the invertibility of $B, G^{\prime}(\bar{z})$ is nonsingular.

(d) This follows from Proposition 6 and Theorem 1

Example 3 Let $V=\mathcal{S}^{n}$, the Euclidean Jordan algebra of all $n \times n$ real symmetric matrices with $\langle X, Y\rangle:=\operatorname{trace}(X Y)$ and $X \circ Y:=X Y+Y X$. Here, 
$\mathcal{S}_{+}^{n}$ is the 'semidefinite cone' consisting of positive semidefinite matrices in $\mathcal{S}^{n}$. We write $X \succeq 0$ and $X \succ 0$, respectively, to denote elements of $V_{+}$and its interior. Let $A$ be an $n \times n$ real matrix which is positive stable (which means that every eigenvalue of $A$ has positive real part). Then, the Lyapunov transformation $X \mapsto A X+X A^{T}$ is a $\mathbf{P}$-transformation on $\mathcal{S}^{n}$ 7. Consequently, for any $W \succ 0$, the following system has a solution:

$$
X \succ 0, Y \succ 0, X \circ Y=W \text {, and } Y=A X+X A^{T}
$$

While the existence of an $X \succ 0$ with $A X+X A^{T} \succ 0$ is already covered in the Lyapunov theory of dynamical systems, what is new here is that such an $X$ can be found satisfying an additional condition $X \circ\left(A X+X A^{T}\right)=W$ where $W \succ 0$ is arbitrary! One can make statements similar to the above for the Stein transformation $X \mapsto X-B X B^{T}$, where $B$ is an $n \times n$ real Schur stable matrix (which means that every eigenvalue of $B$ has absolute value less than one) 7 .

\section{P-pairs over $\mathbb{R}^{n}$}

We now consider $V=\mathbb{R}^{n}$ and prove a uniqueness result.

Theorem 3 Let $V=\mathbb{R}^{n}$. Then, the following statements are equivalent:

(a) $\{A, B\}$ is a $\mathbf{P}$-pair.

(b) $\operatorname{wHLCP}(A, B, w, q)$ has a unique solution for every $(w, q) \in \mathbb{R}_{+}^{n} \times \mathbb{R}^{n}$.

(c) $\operatorname{HLCP}(A, B, q)$ has a unique solution for every $q \in \mathbb{R}^{n}$.

Proof $(a) \Rightarrow(b)$ : The solvability of $\operatorname{whLCP}(A, B, w, q)$ has been addressed in the previous result. We now prove uniqueness. Suppose that $\left(x_{1}, y_{1}\right)$ and $\left(x_{2}, y_{2}\right)$ are any two solutions of $\operatorname{wHLCP}(A, B, w, q)$, i.e.,

$$
\left\{\begin{array} { l } 
{ x _ { 1 } \geq 0 , y _ { 1 } \geq 0 } \\
{ x _ { 1 } * y _ { 1 } = w } \\
{ A x _ { 1 } + B y _ { 1 } = q }
\end{array} \quad \text { and } \quad \left\{\begin{array}{l}
x_{2} \geq 0, y_{2} \geq 0 \\
x_{2} * y_{2}=w \\
A x_{2}+B y_{2}=q
\end{array}\right.\right.
$$

As $w \geq 0$, let

$$
\alpha:=\left\{i: w_{i}>0\right\} \quad \text { and } \quad \beta:=\left\{i: w_{i}=0\right\}
$$

Then,

$$
\begin{aligned}
& \left(x_{1} * y_{1}\right)_{i}=\left(x_{2} * y_{2}\right)_{i}=w_{i}>0, \quad \forall i \in \alpha, \\
& \left(x_{1} * y_{1}\right)_{i}=\left(x_{2} * y_{2}\right)_{i}=w_{i}=0, \quad \forall i \in \beta .
\end{aligned}
$$


Now considering the componentwise product (only) over the $\alpha$ indices, we have

$$
\begin{aligned}
& \left(x_{1}-x_{2}\right) *\left(y_{1}-y_{2}\right) \\
& =x_{1} * y_{1}+x_{2} * y_{2}-x_{1} * y_{2}-x_{2} * y_{1} \\
& =x_{1} * y_{1}+x_{1} * y_{1}-x_{1} *\left(\frac{x_{1} * y_{1}}{x_{2}}\right)-x_{2} * y_{1} \\
& =\left(x_{1}-x_{2}\right) * y_{1}+\left(\frac{x_{1} * y_{1}}{x_{2}}\right) *\left(x_{2}-x_{1}\right) \\
& =\left(x_{2}-x_{1}\right) *\left(\frac{x_{1} * y_{1}}{x_{2}}-y_{1}\right) \\
& =-\frac{y_{1}}{x_{2}}\left(x_{1}-x_{2}\right)^{2} \\
& \leq 0 .
\end{aligned}
$$

And over the $\beta$ indices,

$$
\begin{aligned}
& \left(x_{1}-x_{2}\right) *\left(y_{1}-y_{2}\right) \\
& =x_{1} * y_{1}+x_{2} * y_{2}-x_{1} * y_{2}-x_{2} * y_{1} \\
& =0+0-x_{1} * y_{2}-x_{2} * y_{1} \\
& \leq 0
\end{aligned}
$$

Therefore, we have

$$
\begin{aligned}
& \left(x_{1}-x_{2}\right) *\left(y_{1}-y_{2}\right) \leq 0, \\
& A\left(x_{1}-x_{2}\right)+B\left(y_{1}-y_{2}\right)=0 .
\end{aligned}
$$

As $\{A, B\}$ is a $\mathbf{P}$-pair and vectors in $\mathbb{R}^{n}$ always operator commute, we see that $x_{1}=x_{2}$ and $y_{1}=y_{2}$. Thus we have uniqueness of solution in any ${ }_{\mathrm{wHLCP}}(A, B, w, q)$.

$(b) \Rightarrow(c)$ : This is obvious by taking $w=0$.

$(c) \Rightarrow(a)$ : Suppose $(x, y) \neq(0,0)$ with $x \circ y \leq 0$ and $A x+B y=0$. For $q:=A x^{+}+B y^{+}=A x^{-}+B y^{-}$, we see that $\left(x^{+}, y^{+}\right)$and $\left(x^{-}, y^{-}\right)$are two distinct solutions of $\operatorname{HLCP}(A, B, q)$. This contradicts condition $(c)$.

We remark that such a uniqueness result may not prevail over general Euclidean Jordan algebras even for $\mathbf{P}$-transformations, see the remarks following Theorem 14 in [7.

The above result, especially Item $(c)$, allows us to connect $\mathbf{P}$-pairs to the socalled $\mathbf{W}$ property for a pair of matrices, see [16].

Concluding Remarks. In this paper, we have presented some existence and uniqueness results for weighted horizontal linear complementarity problems over Euclidean Jordan algebras. These are established for $\mathbf{R}_{0}$-pairs of linear transformations satisfying a (nonzero) degree condition. The novelty here is the use of 'weighted' Fischer-Burmeister map and degree theory techniques. We hope to consider applications, algorithms, and non $\mathbf{R}_{0}$-pairs in a future study. 
Acknowledgements The work of the first author is supported by the National Natural Science Foundation of China (No. 11401126) and Guangxi Natural Science Foundation (Nos. 2016GXNSFBA380102, 2014GXNSFFA118001), China. The third author was supported by Loyola Summer Research Grant 2017.

\section{References}

1. Anstreicher, K.M.: Interior-point algorithms for a generalization of linear programming and weighted centering. Optim. Methods Softw. 27(4-5), 605-612 (2012)

2. Cottle, R.W., Pang, J.-S., Stone, R.: The Linear Complementarity Problem. Academic Press, Boston (1992)

3. Eisenberg, E., Gale, D.: Consensus of subjective probabilities: the pari-mutuel method. Ann. Math. Statist. 30, 165-168 (1959)

4. Facchinei, F., Pang, J.S.: Finite-dimensional variational inequalities and complementarity problems. Vol. I, Springer, New York (2003)

5. Faraut, J., Koranyi, A.: Analysis on Symmetric Cones. Oxford University Press, New York (1994)

6. Gowda, M.S.: Applications of degree theory to linear complementarity problems. Math. Oper. Res. 18, 868-879 (1993)

7. Gowda, M.S., Sznajder, R., Tao, J.: Some P-properties for linear transformations on Euclidean Jordan algebras. Special issue on Positivity, Linear Algebra Appl. 393, 203-232 (2004)

8. Kojima, M., Mizuno, S., Noma, T.: Limiting behavior of trajectories generated by a continuation method for monotone complementarity problems. Math. Oper. Res. 15(4), 662-675 (1990)

9. Kojima, M., Megiddo., Noma, T., Yoshise, A.: A Unified Approach to Interior Point Algorithms for Linear Complementarity Problems. Lecture Notes in Computer Science 538, Springer-Verlag, Berlin (1991)

10. Lloyd, N.G.: Degree Theory. Cambridge University Press, London (1978)

11. Ortega, J.M., Rheinboldt, W.C.: Iterative Solutions of Nonlinear Equations in Several Variables, Academic Press, New York (1970)

12. Ouellette, D.V.: Schur complements and statistics. Linear Algebra Appl. 36, 187-295 (1981)

13. Potra, F.A.: Weighted complementarity problems - a new paradigm for computing equilibria. SIAM J. Optim. 22(4), 1634-1654 (2012)

14. Potra, F.A.: Sufficient weighted complementarity problems. Comput. Optim. Appl. 64: 467-488 (2016)

15. Sznajder, R.: Degree-theoretic analysis of the vertical and horizontal linear complementarity problems, PhD Thesis, University of Maryland Baltimore County (1994)

16. Sznajder, R., Gowda, M.S.: Generalizations of $P_{0},(\mathrm{P})$-properties; extended vertical and horizontal LCPs. Linear Algebra Appl. 223/224, 695-715 (1995)

17. Ye, Y.: A path to the Arrow-Debreu competitive market equilibrium. Math. Program. 111(1-2, Ser. B), 315-348 (2008)

18. Yoshise, A.: Interior point trajectories and a homogeneous model for nonlinear complementarity problems over symmetric cones. SIAM J. Optim. 17, 1129-1153 (2006) 